\title{
Notas críticas respecto del Acuerdo de París sobre el cambio climático $^{+}$
}

\author{
JAN-DAVID GELLES* \\ Pontificia Universidad Católica del Perú \\ jgelles@pucp.edu.pe \\ https://doi.org/10.18800/rcpg.201601.006
}

\section{RESUMEN}

En el Acuerdo de París — por primera vez- se plantea un objetivo cuantitativo de estabilización de la temperatura media global, se introduce un mecanismo de coordinación global para alcanzar dicho objetivo y se tiene la ambición de universalizar a todos los países las exigencias de reducción de los gases de efecto invernadero. Sin embargo, podría existir una inconsistencia temporal entre el objetivo declarado y el mecanismo contemplado para implementarlo por razón de la falta de un régimen de sanciones. Las promesas de ayuda financiera a los países afectados por el cambio climático constituyen un rescate con dinero público de las industrias carbonífera, petrolífera y gasífera.

Palabras clave: Acuerdo de París, cambio climático, capitalismo.

Critical notes regarding the Paris agreement on climate change.

\begin{abstract}
In the Paris Agreement - for the first time - a quantitative objective for stabilizing the global average temperature is proposed, a global coordinating mechanism is introduced to achieve this objective and the ambition is to universalize to all countries the reduction requirements of greenhouse gases. However, there could be a temporary inconsistency between the stated objective and the mechanism contemplated to implement it because of the lack of a sanctions regime. The promises of financial help to countries affected by climate change constitute a bailout with public money of the coal, oil and gas industries.
\end{abstract}

Key words: Paris Agreement, climate change, capitalism.

\footnotetext{
* Profesor del curso de maestría de Introducción a la Economía del Sector Público de la Escuela de Gobierno y Políticas Públicas de la Pontificia Universidad Católica del Perú. El autor agradece los comentarios de la profesora Julissa Castro sobre una primera versión de este artículo.

+ Este artículo está basado en una charla dictada por el autor por invitación de la Fundación Academia Diplomática del Perú a los alumnos del Curso de Verano 2016.

Recibido el 11 de abril de 2016; aceptado el 28 de septiembre de 2016.
} 



\section{INTRODUCCIÓN}

En la $21^{\text {ava }}$ Conferencia de las Partes de la Convención Marco de las Naciones Unidas sobre el Cambio Climático (CMNUCC) celebrada en París, Francia, del 30 de noviembre de 2015 al 11 de diciembre de 2015 se aprobó el Acuerdo de París. ${ }^{1}$

El Acuerdo de París consiste de un preámbulo y 29 artículos, y en el mismo se contempla que su entrada en vigor tendrá lugar después de 30 días contados desde la fecha en que no menos de 55 Partes de la CMNUCC — cuyas emisiones estimadas representen globalmente un 55\% del total de las emisiones mundiales de gases de efecto invernadero- hayan depositado sus instrumentos de ratificación, aceptación, aprobación o adhesión ante el Secretario General de la Organización de las Naciones Unidas.

Según el Secretariado Ejecutivo de la CMNUCC, el Acuerdo de París entraría en vigor el día 4 de noviembre de 2016, en vista de la rápida e inesperada velocidad de firma y ratificación del mismo $^{2}$ (y en efecto, eso fue lo que ocurrió). ${ }^{3}$

De importancia, la lista oficial de países que firmaron el Acuerdo de París -en una ceremonia llevada a cabo en la sede central de la Organización de las Naciones Unidas en Nueva York el 22 de abril de 2016 - incluyó a la República Popular China y a los Estados Unidos de Norteamérica (se puede revisar la lista completa de países que firmaron y posteriormente ratificaron el Acuerdo de París accediendo a la página electrónica correspondiente del United Nations Treaty Collection). ${ }^{4}$

En el Preámbulo del Acuerdo de París se reafirman principios fundamentales que deberán guiar las acciones colectivas para enfrentar el problema de cambio climático, tales como los principios de la equidad y de las responsabilidades comunes pero diferenciadas; y se reconoce la necesidad de una respuesta "progresiva y eficaz" sobre la amenaza que representa el cambio climático.

En el Acuerdo de París se plantea por primera vez un objetivo de estabilización de la temperatura media global porque se busca «mantener el aumento de la

\footnotetext{
En adelante se hará referencia al preámbulo y a los artículos del Acuerdo de París omitiendo la fuente, la cual puede consultarse en la bibliografía como UNFCCC (2015).

2 Véase el comunicado "; Celebre con nosotros la entrada en vigor del Acuerdo de París!» (NEWSROOM-UNFCCC 2016a).

3 Véase el comunicado "El Acuerdo de París entra en vigor dando luz verde a la acción mundial frente al cambio climático", (NEWSROOM-UNFCCC 2016b).

4 A la fecha de revisión del estado de ratificación del Acuerdo de París, 86 países ya eran partes del acuerdo. Perú firmó el acuerdo en la ceremonia del 22 de abril de 2016 y ratificó el mismo el 25 de julio del mismo año (UNTC 2016).
} 
temperatura media mundial muy por debajo de los $2^{\circ} \mathrm{C}$ ccon respecto a los niveles preindustriales»; ese objetivo no aparece en el Protocolo de Kyoto de 1997 y su formulación específica tuvo como objetivo satisfacer durante la negociación a los pequeños Estados insulares que ya están padeciendo inundaciones de sus costas (dichos estados habían demandado un objetivo de estabilización de la temperatura media global de $1.5^{\circ} \mathrm{C}$ ).

En realidad, y considerando que en el año 2015 la temperatura media global alcanzó $1^{\circ} \mathrm{C}$ ppor encima de las temperaturas preindustriales — por primera vez desde que se tienen mediciones confiables ${ }^{5}$ que ese incremento se puede volver la norma si continúa la tendencia del calentamiento global ${ }^{6}$, el Acuerdo $^{\circ}$ de París implica un objetivo de limitación del incremento de la temperatura media global en relación a la temperatura presente en $1^{\circ} \mathrm{C}$.

Adicionalmente, en el Acuerdo de París se ha establecido que el "punto máximo» o pico de las emisiones de gases de efecto invernadero de los países desarrollados será anterior al pico de los países en desarrollo, en consistencia con los principios de equidad anteriormente mencionados.

Según el Acuerdo de París, y para cumplir con el objetivo de estabilización de la temperatura media global, las emisiones netas globales de gases de efecto invernadero deberán ser cero «en la segunda mitad de siglo»; es decir, se transitaría hacia una economía con baja intensidad en el uso del carbono dentro de algunas décadas.

Hay que resaltar que la propuesta de reducción de los gases de efecto invernadero plasmada en el Acuerdo de París - y en contraste con el Protocolo de Kyoto - no excluye a los países en desarrollo; por lo tanto, aquellos países que son grandes emisores de dióxido de carbono $\left(\mathrm{CO}_{2}\right)$ yy otros gases de efecto invernadero y a la vez países en desarrollo pueden quedar vinculados legalmente a la referida propuesta, tales como la República Popular China, la República de la India, la República Federativa del Brasil, la República de Indonesia, el Reino de Arabia Saudita, los Estados Unidos de México, la República Islámica de Irán, entre otros. ${ }^{7}$

\section{ANÁLISIS}

\footnotetext{
Véase «Global temperatures set to reach $1^{\circ} \mathrm{C}$ marker for first time» (Met Office 2015).

6 El ańo 2015 ha sido, además, declarado como el ańo más caluroso desde 1850, y en consecuencia continúa la tendencia anómala en las temperaturas medias globales observada desde la década del 2000. Véase «2015: the warmest year on record, say scientists» (Met Office 2016).

7 Véase «List of Countries by Carbon Dioxide Emissions» (World Bank 2016).
} 
En el Gráfico 1 se puede apreciar a modo de ilustración las trayectorias de las emisiones de gases de efecto invernadero de los países desarrollados y de los países en desarrollo (nótese que los tramos de subida de las dos "colinas» son menos empinados que los tramos de bajada para indicar progresividad).

Tal como lo estipula el Acuerdo de París, el pico máximo de las emisiones de los países desarrollados es anterior al pico de máximo de las emisiones de los países en desarrollo y, adicionalmente, en ambos casos, se produce una caída de las emisiones hasta llegar a cero en alguna fecha no determinada después del año 2050 («economía descarbonizada»).

Gráfico 1. Trayectoria de las emisiones de $\mathrm{CO}_{2}$ según el acuerdod de París

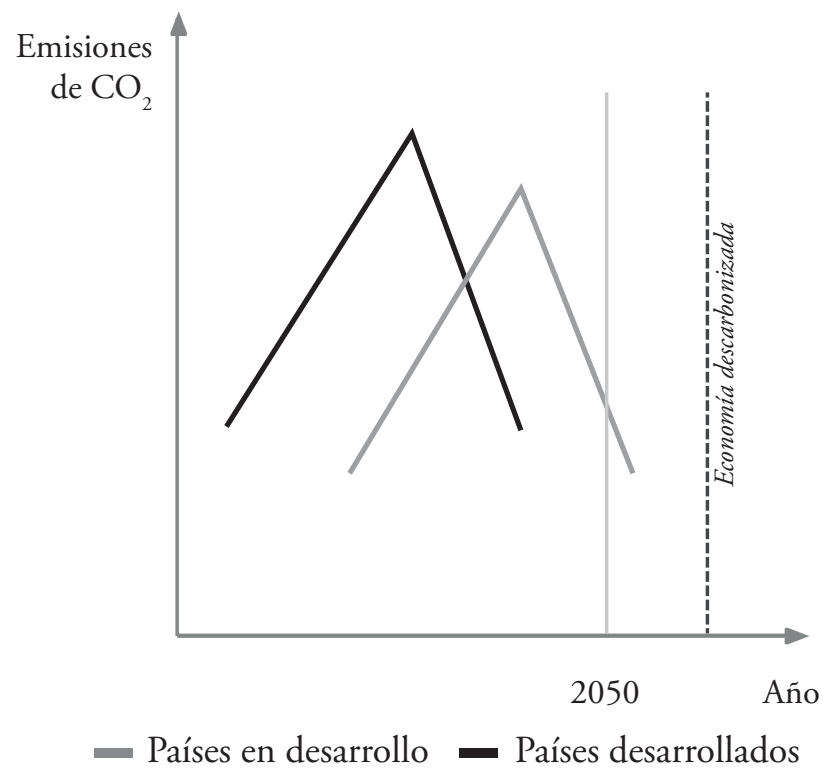

Elaboración propia.

Obsérvese que el cúmulo de las emisiones de gases de efecto invernadero de los países desarrollados y de los países en desarrollo está dado por la sumatoria de las emisiones de cada grupo de países desde el presente y hasta esa fecha no determinada después del año 2050; vale decir, ese cúmulo de emisiones es igual a la sumatoria de las áreas debajo de las curvas respectivas.

En términos algebraicos, $\sum \mathrm{e}_{1 \mathrm{t}}+\sum \mathrm{e}_{2 \mathrm{t}}=\overline{\mathrm{e}}$, donde elt representa el nivel de emisiones de gases de efecto invernadero de los países desarrollados en el tiempo t, $e_{2 t}$ representa el nivel de emisiones de los países en desarrollo en t, è 
representa el máximo acumulativo de emisiones de gases de efecto invernadero consistente con el objetivo de estabilización del clima global.

De acuerdo a estimaciones científicas publicadas por el Met Office del Reino Unido, se podrían emitir 2,900 gigatoneladas de $\mathrm{CO}_{2}\left(\mathrm{GtCO}_{2}\right)$ para mantener el incremento de la temperatura media global por debajo de los $2^{\circ} \mathrm{C}$ con una probabilidad mayor al 66\%; empero, y considerando que ya se han emitido alrededor de 2,000 $\mathrm{GtCO}_{2}$, el cúmulo máximo adicional de emisiones de $\mathrm{CO}_{2}$ sería de $900 \mathrm{GtCO}_{2}{ }^{8}$

Por lo tanto, è $\leq 900 \mathrm{GtCO}_{2}$, vale decir, el cúmulo máximo adicional de emisiones de $\mathrm{CO}_{2}$ queda acotado en el límite superior por dicha cantidad (nótese que no necesariamente hay igualdad estricta porque hay una diferencia en el tiempo entre la producción del estimado científico y la eventual entrada en vigor del Acuerdo de París).

Ese límite teórico, acotado ya numéricamente, define las condiciones de la pugna política entre y dentro de los dos grupos de países - ricos y pobres - en futuras negociaciones climáticas; cada país pugnará por cuestiones económicas por recibir una asignación elevada de esa cantidad limitada de emisiones de dióxido de carbono dado que tanto la producción de bienes como el transporte de los mismos son intensivos en el uso de carbono.

No obstante, y a pesar de la disponibilidad de estimados científicos, el Acuerdo de París no establece ninguna magnitud respecto al cúmulo máximo adicional de emisiones de $\mathrm{CO}_{2}$ ni tampoco fija pautas acerca de cómo se dividiría esa magnitud entre los países desarrollados y los países en desarrollo, y dentro de cada uno de esos grupos; en otros términos, no se ha establecido un presupuesto global de emisiones de carbono ni una fórmula para su distribución entre países. ${ }^{9}$

Por otro lado, en el Acuerdo de París sí se contempla un mecanismo de «balance mundial» para determinar el avance colectivo en el cumplimiento de los objetivos de reducción de las emisiones de gases de efecto invernadero a largo plazo.

Ese mecanismo es crucial porque en el Acuerdo de París se establecen "contribuciones determinadas a nivel nacional" para reducir progresivamente las emisiones de gases de efecto invernadero; $y$, en ausencia de un mecanismo de coordinación, no habría ninguna garantía de una armonización entre los planes

\footnotetext{
Véase «Global temperatures set to reach $1^{\circ} \mathrm{C}$ marker for first time» (Met Office 2015).

9 Agradezco a uno de los árbitros por la sugerencia de utilizar aquí el término presupuesto de emisiones de carbono.
} 
determinados nacionalmente y los requerimientos globales; sin embargo, el mecanismo de balance mundial tiene carácter cooperativo.

En consecuencia, no necesariamente tiene por qué haber consistencia entre la trayectoria temporal de las emisiones y los objetivos de estabilización de la temperatura media global planteados en el Acuerdo de París (las dos «colinas» del Gráfico 1 podrían ser muy altas). ${ }^{10}$

En lo que respecta a las pérdidas y daños, el Acuerdo de París refuerza el Mecanismo Internacional de Varsovia para las Pérdidas y los Daños relacionados con las Repercusiones del Cambio Climático; vale decir, se opta por reforzar un mecanismo de carácter cooperativo en vez de uno de carácter contencioso y centrado en la compensación, reparación y sanción a las industrias del carbón, petróleo y gas (estas industrias desaparecerían virtualmente cuando la economía mundial transite hacia fuentes de energía bajas en carbono).

Dado que en el Acuerdo de París se renueva el compromiso de los países desarrollados de aportar recursos financieros públicos para financiar la adaptación, mitigación, transferencia de tecnología y otras inversiones para enfrentar el cambio climático, se puede afirmar entonces que el enfoque cooperativo adoptado respecto de las pérdidas y daños, representa un rescate de las industrias carbonífera, petrolífera y gasífera con dinero de los contribuyentes de impuestos; en otras palabras, se ha buscado socializar las pérdidas y daños reales y potenciales de esas industrias, todavía muy poderosas, pero destinadas a un declive definitivo en el largo plazo.

En el Acuerdo de París no hay montos específicos de dinero comprometidos para financiar las inversiones arriba mencionadas; $y$, por lo tanto, es imposible compararlos con los montos —en magnitudes de varios billones de dólaresde los rescates con dinero público a la banca, seguros, automotrices y otras industrias de años recientes. ${ }^{11}$

Tampoco se contempla en el Acuerdo de París la fijación de un impuesto global que sea proporcional al contenido de carbono de los bienes y servicios, que penalizaría a las industrias de combustibles fósiles y permitiría transferir recursos económicos para financiar los bienes públicos necesarios para enfrentar el fenómeno del cambio climático.

\footnotetext{
10 Véase, para una conclusión similar, el artículo «Paris Climate Talks: Analysis of the Final Agreement» (Bennet 2015).

11 No obstante, como parte de compromisos financieros asumidos anteriormente, el Secretariado Ejecutivo de la CMNUCC hace referencia a un monto de 100,000 millones de dólares en ayudas oficiales que permitiría un apalancamiento privado para alcanzar entre 5 y 7 billones de dólares para hacer posible la transformación a nivel global. Véase «El histórico Acuerdo de París, a punto de entrar en vigor» (NEWSROOM-UNFCCC 2016c).
} 
Esa medida impositiva es consistente con el principio económico de que «el que contamina paga», y fue propuesta por Joseph E. Stiglitz en su ensayo Cómo hacer que funcione la globalización, en los siguientes términos:

"La forma de hacerlo es imponer a todos los países del mundo una tasa común sobre las emisiones de dióxido de carbono (es decir, gravar el factor externo de las emisiones) o, de forma alternativa, imponer un gravamen al petróleo, carbón y el gas que refleje las emisiones que estos combustibles generan" (Stiglitz 2006: 235).

Considerando que un país se pondría en desventaja comercial frente a otro de imponer de manera unilateral un impuesto proporcional al contenido de carbono de los bienes y servicios, hubiese sido necesario que esa medida se introduzca coordinadamente a nivel global.

\section{Comentarios}

Es evidente que hay cierto progreso en la decisión de fijar un objetivo cuantitativo para la estabilización del clima global y además en la pretensión de que los esfuerzos de reducción de las emisiones de los gases de efecto invernadero sean ahora universales; sin embargo, no se desprende del Acuerdo de París que se vaya a declarar una emergencia climática y una acción progresiva para resolver el problema del calentamiento global no parece justificada.

De acuerdo al sitio 350.org, fundado por el medioambientalista norteamericano Bill McKibben, la concentración atmosférica segura de C02 es de $350 \mathrm{ppm}$ y la concentración observada supera ya las $400 \mathrm{ppm} .{ }^{12}$ Por lo tanto, estamos ya por encima del nivel seguro, y ese nivel de concentración seguro recibe respaldo de científicos destacados sobre el cambio climático como James Hansen y Rajendra Pachauri. ${ }^{13}$

Tampoco está garantizada la eficacia del esfuerzo planteado en el Acuerdo de París para armonizar los objetivos de estabilización de la temperatura media global en $+2^{\circ} \mathrm{C}$ en relación con las temperaturas medias preindustriales y los objetivos nacionales de mitigación, porque se ha optado por un mecanismo de coordinación central de carácter voluntario y exento de sanciones.

Hay además implícito en el Acuerdo de París un rescate con dinero de los contribuyentes de impuestos a la industria de los combustibles fósiles, dado que se manifiesta un compromiso de transferir dinero público a los países

\footnotetext{
12 De acuerdo a datos preliminares divulgados el 4 de octubre de 2016 en la página web co2.earth, la concentración atmosférica de CO2 es de 401.01 ppm (co2.earth 2016).

13 Véase el sustento científico propuesto por la organización 350.org en su página web.
} 
afectados por el cambio climático, pero los daños y pérdidas no se recuperarán demandando a los responsables directos.

También es cierto que se podrían introducir enmiendas posteriores al Acuerdo de París, o protocolos adicionales al mismo, si persisten las acciones del movimiento por la justicia climática y la presión de los ciudadanos en la dirección indicada en el presente artículo.

En ese sentido, hay que señalar que existen acuerdos internacionales que son posteriores a la aprobación del Acuerdo de París y que tienen incidencia sobre el objetivo de estabilización de la temperatura media global por debajo de los $2{ }^{\circ} \mathrm{C}$ : en una enmienda al Protocolo de Montreal relativo a las Sustancias que Agotan la Capa de Ozono, aprobada en la $28^{\text {ava }}$ Reunión de las Partes celebrada en Kigali, Ruanda el 15 de octubre del 2016, se acordó una reducción drástica de unos potentes gases de efecto invernadero denominados hidrofluorocarbonos (HFCs) ${ }^{1415}$; y en la $39^{\text {ava }}$ Asamblea de la Organización de Aviación Civil Internacional (OACI) celebrada en Montreal, Canadá el 8 de febrero del 2016 se acordó un plan para compensar las emisiones de CO2 de la aviación comercial. ${ }^{16}$

Como se mencionó en una nota al pie de página en la sección de Introducción, nuestro país ya firmó y ratificó el Acuerdo de París; por lo tanto, el anuncio que hizo el Perú a la Secretaría Ejecutiva de la CMNUCC en la Asamblea General de la ONU, llevada a cabo en Nueva York el 26 de setiembre de 2015, respecto de la contribución prevista y determinada a nivel nacional para reducir las emisiones de gases de efecto invernadero, tendrá carácter de un compromiso internacional firme con la prevista entrada en vigor del Acuerdo de París.

En otros términos, las contribuciones nacionales comunicadas por el Perú y otros países a la Secretaría Ejecutiva de la CMNUCC no son vinculantes hasta la entrada en vigor del Acuerdo de París (dichas contribuciones se podrían modificar con posterioridad, aunque solo al alza).

Nuestro país ha previsto una reducción del $30 \%$ respecto de las emisiones de gases de efecto invernadero proyectadas para el año 2030, comparada a la tendencia actual de las emisiones nacionales. Y dichas acciones de reducción serán desarrolladas principalmente a través del sector forestal, y dependerán críticamente de su eficacia en ese sector de la economía (pero además se

\footnotetext{
14 Véase «Countries Agree to Curb Powerful Greenhouse Gases in Largest Climate Breakthrough since Paris» (UNEP 2016).

15 Dichos gases son benignos para la capa de ozono y se convirtieron en alternativas para los clorofluorocarbonos (CFCs). Véase «Phasing Down HFCs, the Climate Low-Hanging Fruit» (Espinosa y Molina 2016).

16 Véase «U.N. Group Agrees to Aircraft Standards to Cut CO2 Emissions» (Reuters 2016).
} 
desarrollarán acciones en los sectores industrial, energético, agrícola, así como en el sector transporte y residuos). ${ }^{17}$

En el Perú una parte muy importante de la generación de electricidad proviene de fuentes limpias y renovables; a saber, las plantas hidroeléctricas y aquellas fuentes de energía no convencionales: en nuestro país la participación de las unidades hidráulicas en la producción total de energía eléctrica fue de $48 \%$ en el año 2015 y la participación de las unidades solares y eólicas fue de $2 \%$ en ese mismo año (cifras preliminares). ${ }^{18}$

Un $50 \%$ de la generación de energía eléctrica ya proviene de fuentes renovables, convencionales o no convencionales, según estadísticas oficiales peruanas; ${ }^{19}$ sin embargo, la participación de las fuentes no convencionales es muy baja y es necesario diversificar las fuentes de generación eléctrica, porque el retroceso de los glaciares andinos tendrá un efecto negativo sobre la producción de energía hidroeléctrica en Lima y otras ciudades de la costa del Perú y además afectará el suministro de agua cruda para potabilización.

Alemania es otro país que está haciendo esfuerzos reconocidos para transformar su sistema de generación de energía eléctrica: en ese país el 30\% de la generación de energía proviene del viento, la biomasa, el sol y el agua al año 2015 (no incluye la variedad de carbón altamente contaminante que Alemania extrae y exporta a terceros países). ${ }^{20}$

Sin embargo, la implementación efectiva del Acuerdo de París dependerá críticamente de las acciones que tomen los Estados Unidos de Norteamérica bajo la presidencia del republicano Donald J. Trump, a partir de la asunción del mando programada para el 20 de enero de 2017.

El discurso de negación sobre el cambio climático que hizo Donald J. Trump en la campaña electoral de su país, su promesa de «cancelar»" el Acuerdo de París, la designación de un cabildero de la industria del carbón para liderar la transición en la Agencia Gubernamental de Protección Ambiental (EPA, por

\footnotetext{
17 Véase el anuncio del Ministerio del Ambiente sobre la contribución prevista y determinada a nivel nacional y el documento que la sustenta (Ministerio del Ambiente 2015).

18 Véase «Evolución de Indicadores del sector eléctrico 1995-2015» (Ministerio de Energía y Minas 2015).

19 Cabe recordar que en el marco de la COP20 realizada en nuestro país, Manuel Pulgar-Vidal Otálora, Ministro del Ambiente, resaltó los logros del Perú por haber alcanzado esa cifra, de cerca de $50 \%$ de producción de energía eléctrica de fuentes renovables, como porcentaje del total de producción de energía eléctrica.

20 Véase los progresos realizados por ese país en la conversión de su sistema energético en «Rekordjahr für Erneuerbare in Deutschland» (Deutsche Welle 2016).

21 Sigue una transcripción de un discurso de campańa electoral de Donald J. Trump emitido por el programa televisivo World News Today (una reproducción considerando que las elecciones presidenciales ya habían terminado): "We are going to cancel the Paris Climate Agreement, and stop.... all payments of US tax dollars to UN Climate Programs" (BBC World 2016).
} 
sus siglas en inglés), y la elección de un Congreso dominado por el Partido Republicano son señales desalentadoras. ${ }^{22}$

\section{REFLEXIONES}

Para finalizar, hay que concluir que el fenómeno de cambio climático tiene sus raíces en un sistema económico dominado por grandes corporaciones capitalistas y que estas operan bajo una lógica «racional» de exteriorización de costos, y además bajo la necesidad permanente de ampliar el tamaño de sus mercados, en perjuicio del medioambiente.

Como lo ha expresado Naomi Klein en Esto lo cambia todo, su notable libro sobre el cambio climático:

[...] la razón real por la que no estamos reaccionando a la altura de lo que exige el momento climático actual es que las acciones requeridas para ello ponen directamente en cuestión nuestro paradigma económico dominante (Klein 2015: 88).

Hay que señalar entonces que las acciones para contrarrestar el cambio climático en curso y prevenir escenarios catastróficos se deben centrar en el sistema económico; en términos lógicos, habría que reformarlo u optar por una transición a un nuevo sistema económico.

Es cierto, al mismo tiempo, que los individuos — insertados en un sistema económico- también pueden tomar acciones para prevenir un cambio climático catastrófico.

Asimismo, hay que recordar que si el sistema económico condiciona al sistema político para impedir acciones para prevenir un cambio climático catastrófico, los retos que se plantean son también políticos.

Finalmente, hay que resaltar que la humanidad enfrenta una crisis ecológica que no se reduce al problema de cambio climático, sino que abarca otros problemas que son graves, irreversibles y de carácter global.

22 Véase «Trump's Climate Contrarian: Myron Ebell Takes On the E.P.A» (New York Times 2016). 


\section{Bibliografía}

BBC World (2016). World News Today. Programa emitido el 14 de noviembre del 2016 a las 14:00 horas.

Bennet, Craig (2015). «Paris Climate Talks: Analysis of the Final Agreement». Friends of the Earth. London, 12 December. Consulta: 18 de enero de 2016. http://www.foe.co.uk/blog/ paris-climate-talks-analysis-final-agreement

Detusche Welle (2016). «Rekordjahr für Erneuerbare in Deutschland». Deutsche Well: Wissen \& Umwelt. 7 de enero. Consulta: 7 de enero de 2016. http://www.dw.com/de/ rekordjahr-f\%C3\%BCr-erneuerbare-in-deutschland/a-18966221

Espinosa, Patricia y Mario Molina (2016). «Phasing Down HFCs, the Climate Low-Hanging Fruit». Newsroom-UNFCCC. 10 de octubre. Consulta: 28 de octubre de 2016. http://newsroom.unfccc.int/unfccc-newsroom/phasing-down-hfcs-the-climate-s-low-hanging-fruit/

Klein, Naomi (2015). Esto lo cambia todo: el capitalismo contra el clima. Barcelona: Paidos Ibérica.

MET Office (2015). «Global temperatures set to reach $1^{\circ} \mathrm{C}$ marker for first time». Met Office. United Kingdom. 9 de noviembre. Consulta: 20 de enero de 2016. http://www.metoffice.gov. $\mathrm{uk} /$ news/release/archive/2015/one-degree

MET Office (2016). «2015: the warmest year on record, say scientists». Met Office. United Kingdom, 20 de enero. Consulta: 20 de enero de 2016. http:/www.metoffice.gov.uk/news/ releases/archive/2016/2015-global-temperature

Ministerio del Ambiente (2015). «Perú anuncia su Contribución Prevista y Determinada a Nivel Nacional (INDC) en la Asamblea General de las Naciones Unidas de Nueva York». Ministerio del Ambiente, Prensa. Nueva York, 26 de setiembre. Consulta: 5 de julio de 2016. http://www. minam.gob.pe/indcs/2015/09/26/peru-anuncia-su-contribucion-prevista-y-determinada-anivel-nacional-indc-en-la-asamblea-general-de-las-naciones-unidas-de-nueva-york/

Ministerio de Energía y Minas (2015). «Evolución de Indicadores del sector eléctrico 1995-2015». Lima: Misterio de Energía y Minas. Consulta: 4 de enero de 2016. http://www.minem.gob.pe/ archivos/EVOLUCIONES_1995-2015_FINAL-7582kuz.pdf

New York Times (2016). «Trump's Climate Contrarian: Myron Ebell Takes On the E.P.A.». 11 de noviembre. Consulta: 11 de noviembre del 2016. http://www.nytimes.com/2016/11/12/ science/myron-ebell-trump-epa.html?_r=0 , 11 de noviembre del 2016 [11 de noviembre del 2016].

Newsroom-UNFCCC (2016a). "iCelebre con nosotros la entrada en vigor del Acuerdo de París!». 3 de noviembre. Consulta: 24 de octubre de 2016. http://newsroom.unfccc.int/es/ acuerdo-de-paris/celebre-con-nosostros-la-entrada-en-vigor-del-acuerdo-de-paris/

Newsroom-UNFCCC (2016b). «El Acuerdo de París entra en vigor dando luz verde a la acción mundial frente al cambio climático». 4 de noviembre. Consulta: 4 de noviembre de 2016. http://newsroom.unfccc.int/es/noticias/el-acuerdo-de-paris-entra-en-vigor-dando-luz-verde-ala-accion-mundial-frente-al-cambio-climatico/

Newsroom-UNFCCC (2016c). «El histórico Acuerdo de París, a punto de entrar en vigor». 5 de octubre. Consulta: 26 de octubre del 2016. http://newsroom.unfccc.int/es/noticias/ el-historico-acuerdo-de-paris-a-punto-de-entrar-en-vigor/

Reuters (2016). «U.N. Group Agrees to Aircraft Standards to Cut CO2 Emissions». Reuters. Montreal/Washington, 8 de febrero. Consulta: 28 de octubre de 2016. http://www.reuters. com/article/us-climatechange-aviation-idUSKCN0VH1XA

Stiglitz, Joseph E. (2006). Cómo hacer que funcione la globalización. México: Taurus. 
UNTC (2016). "Chapter XXVII». United Nations Treaty Collection. Consulta: 27 de octubre de 2016. https://treaties.un.org/Pages/ViewDetails. aspx?src=TREATY\&mtdsg_no=XXVII-7-d\&chapter=27\&clang=_en

UNFCCC (2015). Aprobación del Acuerdo de París. Convención Marco sobre el Cambio Climático. Naciones Unidas: París, 12 de diciembre. http://unfccc.int/resource/docs/2015/ cop21/spa/109s.pdf

UNEP (2016). "Countries Agree to Curb Powerful Greenhouse Gases in Largest Climate Breakthrough since Paris». United Nations Environment Programme. News Centre. 15 de octubre. Consulta: 28 de octubre de 2016. http://www.unep.org/newscentre/Default.aspx?Doc umentID $=27086 \&$ ArticleID $=36283 \& \mathrm{l}=\mathrm{en}$

World Bank (2016). «CO 2 emissions (kt)». 5 de diciembre del 2016.

http://data.worldbank.org/indicator/EN.ATM.CO2E.KT 